\title{
Urinary isoflavonoid excretion is similar after consuming soya milk and miso soup in Japanese-American women
}

\author{
Gertraud Maskarinec*, Kirsten Watts, Jamie Kagihara, Sandra M. Hebshi and Adrian A. Franke \\ Cancer Research Center of Hawai'i, 1236 Lauhala Street, Honolulu, HI 96813, USA \\ (Received 7 June 2007 - Revised 29 November 2007 - Accepted 30 November 2007 - First published online 14 February 2008)
}

Based on the hypothesis that isoflavones are absorbed more efficiently from fermented than from non-fermented soya foods, we compared the urinary isoflavonoid excretion (UIE) after intake of miso soup or soya milk. We recruited twenty-one women with Japanese ancestry who consumed standardized soya portions containing $48 \mathrm{mg}$ isoflavones. On day 1, half the women consumed soya milk, the other half started with miso soup. On day 3, the subjects ate the other soya food and on day 5, they repeated the first food. Each participant collected a spot urine sample before and an overnight urine sample after soya food intake. All urine samples were analysed for daidzein, genistein and equol using LC-MS and were expressed as nmol/mg creatinine. We applied mixed models to evaluate the difference in UIE by food while including the baseline values and covariates. Relative to baseline, both groups experienced significantly higher UIE after consuming any of the soya foods. We observed no significant difference in UIE when soya milk was compared to miso soup $(P=0 \cdot 87)$ among all women or in the seven equol producers $(P=0 \cdot 88)$. Repeated intake of the same food on different days showed high reproducibility within subjects. These preliminary results indicate similar UIE after consuming a fermented soya food (miso) as compared to a non-fermented soya food (soya milk). Therefore, recommendations favouring fermented soya foods are not justified as long as the intestinal microflora is capable of hydrolysing the isoflavone glucosides from non-fermented soya foods.

Soya: Isoflavones: Urinary isoflavonoid excretion: Equol

Soya foods may protect against chronic diseases ${ }^{(1,2)}$. Isoflavones in soyabeans, i.e. daidzein, genistein and glycitein, are active substances that have chemical structures similar to mammalian oestrogens ${ }^{(3)}$. In food, they are primarily found as $\beta$-glucosides with and without additional malonates and acetate esters $^{(4)}$. Once ingested, these conjugated isoflavones undergo hydrolysis by $\beta$-glucosidases mainly from intestinal bacteria, releasing the principal bioactive aglucone (glucosefree) form that is absorbed, whereas the highly water-soluble glucosides are not absorbed ${ }^{(5-8)}$. Therefore, intestinal bacteria are crucial for the absorption and bioavailability of isoflavones ${ }^{(5,8,9)}$. It is the aglycones (sugar-free forms) that show an affinity for oestrogen receptors and have other non-hormonal effects on the cell machinery ${ }^{(6)}$. The isoflavone daidzein is metabolized to equol and $O$-desmethylangolensin by gut bacteria and excreted predominantly through the urine ${ }^{(10)}$. The ability to produce equol is limited to $30-50 \%$ of the population but whether this metabolic feature results in more beneficial health effects from soya consumption remains uncertain $^{(9,11)}$. The extent of isoflavone metabolism varies among individuals and may be influenced by additional dietary factors ${ }^{(12,13)}$.

Dietary isoflavonoids are specific to soya foods and urinary isoflavonoid excretion (UIE) serves as an excellent marker for the bioavailability of isoflavones ${ }^{(14,15)}$. As a result of their rapid metabolism, urinary appearance of isoflavonoids reflects circulating levels when the timing of specimen collection is accurately considered ${ }^{(8,16,17)}$. Due to micro-organism-induced hydrolysis in fermented soya products, e.g. tempeh and miso, the predominant form of isoflavones in these foods are aglucones. Consuming fermented soya foods was reported to lead to higher levels of absorption and urinary isoflavone recovery in some ${ }^{(18-20)}$ but not in other studies ${ }^{(21-23)}$. We hypothesized that women will excrete more isoflavonoids and produce more equol after consuming equivalent isoflavone amounts in one serving of miso soup than in one serving of soya milk because the aglucone form present in fermented soya products is more bioavailable than the conjugated isoflavones in the non-fermented soya milk.

\section{Methods \\ Participants \\ We recruited a convenience sample through employees, families and friends of the Cancer Research Center of Hawaii. Eligible participants were at least 18 years of age, at least $50 \%$ Japanese ancestry, current residents of Hawaii and free of any known soya allergies. Women with Japanese ancestry were chosen because they were expected to have had previous soya food exposure and a more comparable gut flora than subjects with different ancestries ${ }^{(24)}$. During the}


intervention, none of the participants were taking antibiotics, food supplements or probiotics that could alter the intestinal flora. The study protocol was approved by the Committee on Human Subjects at the University of Hawaii and all subjects gave written informed consent.

\section{Data collection}

Demographic, familial and medical information was collected by questionnaire. Regular soya intake was assessed with a twelve-item questionnaire which elicited the frequency and average serving size of soya food consumed during the last 12 months ${ }^{(25)}$. To obtain a summary score, we multiplied the frequencies of intake for each of the twelve soya foods by the estimated isoflavone content from the food composition database maintained by the Nutrition Support Shared Resource at the Cancer Research Center of Hawaii. Isoflavone concentrations for soya foods in this database were primarily derived from an analysis of representative local foods ${ }^{(4)}$. An additional lifetime questionnaire estimated soya exposure since birth using the following stages: infancy (1 year), childhood (1-9 years), adolescence (10-19 years), early adulthood (20-29 years) and late adulthood $(30+\text { years })^{(26)}$. Participants indicated the annual frequency of four categories of soya foods (tofu; soyabeans and sprouts; soyabean drink or milk; and other soya products) for each period. To obtain a summary score, we multiplied the frequency of intake for each of the four foods by the estimated isoflavone amount and added the total amounts of the four foods in each stage of life to determine an annual intake for each stage.

\section{Intervention and urine collection}

The $6 \mathrm{~d}$ study protocol included three spot urine collections after a soya-free day and three overnight urine collections after consumption of a standardized portion of miso soup or soya milk. According to the HPLC analyses, one serving of soya milk (250 ml Edensoy ${ }^{\circledR}$ Original Organic soya milk) contained $48.3 \mathrm{mg}$ isoflavones as aglycone equivalents which was matched with $31 \mathrm{~g}$ Haccho miso (again determined by HPLC) to be dissolved in one cup of water; $98 \%$ of the isoflavones in miso were aglycones but only $2 \%$ in the soya milk. During and $1 \mathrm{~d}$ before starting the intervention, participants were instructed to abstain from all soya foods other than those provided to them for the study. Half of the participants started with soya milk (group A) as follows. On day 1, at around 18.00 hours, the women collected a spot urine sample, consumed one container of soya milk, and collected all urine thereafter until they got up the next morning (overnight urine). On day 2, participants proceeded with their normal diets. On day 3, subjects followed the same protocol as day 1 , but drank one serving of miso soup. On day 4, participants again returned to their normal diets. On day 5, subjects followed the same protocol as day 1 exactly, collected a spot urine sample at around 18.00 hours, consumed one container of soya milk, and collected all overnight urine. The other half of the participants (group B) followed the same protocol except starting with miso soup (day 1) followed by soya milk (day 3 ) and finally miso soup again (day 5 ). The urine collection containers contained ascorbic and boric acid to prevent bacterial contamination and degradation of analytes.
The samples were stored in refrigerators and transported in chilled coolers. The urine samples were aliquoted into $2 \mathrm{ml}$ vials and stored at $-80^{\circ} \mathrm{C}$ until analysed. Based on the collection times recorded by each subject, we calculated the total number of overnight collection hours.

\section{Urine analysis}

Daidzein, genistein and equol were analysed from urine by LC-MS using a triple quadruple TSQ Ultra system (ThermoFisher, San Jose, CA, USA) with electrospray ionization in negative mode and multiple reaction monitoring ${ }^{(27,28)}$. In brief, triply ${ }^{13} \mathrm{C}$-labelled internal standards of daidzein, genistein and equol (University of St Andrews, UK) were added to $100 \mu \mathrm{l}$ urine and hydrolysed for $1 \mathrm{~h}$ at $37^{\circ} \mathrm{C}$ with glucuronidase and sulphatase (Roche Applied Sciences, Indianapolis, IN, USA) followed by phase separation with diethyl ether ${ }^{(29)}$. The diethyl ether fractions were dried under nitrogen and re-dissolved in a 1:1 mixture of methanolsodium acetate buffer $(0 \cdot 2 \mathrm{M}, \mathrm{pH} 5)$. The extract $(25 \mu \mathrm{l})$ was analysed by LC-MS/MS after separation on a BetaBasic C8-column $(100 \mathrm{~mm} \times 2.1 \mathrm{~mm}$ internal diameter, $3 \mu \mathrm{m})$ coupled to a BetaBasic C8-precolumn $(10 \mathrm{~mm} \times 2.1 \mathrm{~mm}$ internal diameter, $3 \mu \mathrm{m}$; both from ThermoFisher) ${ }^{(27,28,30)}$. The elution was performed with methanol-acetonitrilewater $(10: 10: 80$ to $33: 33: 34)$ in $6 \mathrm{~min}$, holding there for $1 \mathrm{~min}$ before changing in $0.1 \mathrm{~min}$ to the starting mixture for equilibration. Ammonium hydroxide (aqueous $2.5 \%$ at $10 \mu \mathrm{l} / \mathrm{min}$ ) was infused to enhance the signal. Daidzein was monitored using the transitions $(\mathrm{m} / \mathrm{z}) 253.020$ to 222.988 , 207.980 and 131.949; for genistein the transitions were 269.090 to $159.050,133.035$ and 132.032 , and for equol they were 241.130 to $134.950,121.000$ and 118.960 . Limits of quantitation for all analytes were $1.5 \mathrm{nmol} / 1$ for daidzein and genistein and $3.0 \mathrm{nmol} / 1$ for equol for post-intervention samples and half of those values for pre-intervention samples due to differences in concentration steps prior to analysis. Mean intra- and inter-day CV of LC-MS/MS quantitation for daidzein $(422 \mathrm{nmol} / \mathrm{l})$, genistein $(35.9 \mathrm{nmol} / \mathrm{l})$ and equol $(9.9 \mathrm{nmol} / \mathrm{l})$ were $8.3,13.6$ and $2.9 \%$, and $7.3,17.9$ and $3.5 \%$, respectively. Recoveries were $72-88 \%$. Urinary creatinine concentrations were measured with a Roche-Cobas MiraPlus chemistry analyser using a kit from Randox Laboratory (Crumlin, UK) that is based on a kinetic modification of the Jaffe reaction. Limits of quantitation were $<15 \mu \mathrm{mol} / \mathrm{l}$ and the mean inter-assay $\mathrm{CV}$ was $0.8 \%$ at $187 \mu \mathrm{mol} / \mathrm{l}$. The sum of dadzein, genistein and equol was expressed in nmol isoflavonoid/mg creatinine. We also calculated the isoflavonoid excretion as $\mathrm{nmol} / \mathrm{h}$ based on urine weight, hours of urine collection and isoflavonoid concentration in urine.

\section{Statistical analysis}

All data management and statistical analyses were performed using SAS release 9.1 (SAS Institute, Cary, NC, USA). To define equol producer status, we used $0.05 \mathrm{nmol} / \mathrm{mg}$ creatinine as a cut-off value. Two-sample $t$ tests were used to compare study characteristics by group at baseline. Due to their nonnormal distribution, we log transformed the UIE. We examined overall mean differences of UIE after soya milk and miso soup intake in one model that included all six UIE 
values for each woman. This examination was carried out using maximum likelihood estimation of a mixed general linear model that takes into account the covariance structure of the repeated measures within subjects ${ }^{(31)}$. The order (group A or B), day, age, weight and equol producer status were included into the model as covariates. In addition, we computed adjusted least square means of UIE for the three food categories (no soya, soya milk, miso soup).

\section{Results}

We enrolled twenty-one participants with a mean age of 49.4 (range 20-83) years (Table 1). The mean body weight was 58.0 (SD 11.7) kg. All women reported at least $50 \%$ Japanese ancestry. Self-reported isoflavone intake during the previous 12 months varied among the participants with a mean of $20 \cdot 1$ (range $0 \cdot 1-85.6) \mathrm{mg} / \mathrm{d}$. While self-reported early life soya intake was only 0.98 (range $0-7 \cdot 1$ ) servings/d, adult soya intake was 1.7 (range $0 \cdot 2-10 \cdot 2$ ) servings/d. Although group B reported higher soya and isoflavone intake than group A during their previous life, none of the differences was statistically significant. The time during which overnight urine was collected covered a mean of 10.8 (SD 1.5) h and did not differ by group (11.0 v. $10.5 \mathrm{~h} ; P=0.67)$.

The unadjusted mean UIE was $135 \mathrm{nmol} / \mathrm{mg}$ creatinine after first soya milk as compared to $148 \mathrm{nmol} / \mathrm{mg}$ creatinine after first miso soup intake (Fig. 1). When we expressed UIE as an hourly rate ${ }^{(32)}$, the results remained unchanged; the respective medians for soya milk and miso soup were 6.39 and $6.57 \mu \mathrm{mol} / \mathrm{h}$. The correlation between the two UIE values expressed in different units was 0.95. Based on all $6 \mathrm{~d}$, the mean UIE on days after soya food intake were significantly higher than on the days without soya intake $(P<0 \cdot 001$; Fig. 2). In a mixed model that included the baseline UIE values, the difference in UIE after soya milk $v$. miso soup consumption was not significant $(P=0.87)$. The respective adjusted mean UIE for no soya, soya milk and miso soup were $5.7,116.9$ and $111.7 \mathrm{nmol} / \mathrm{mg}$ creatinine, respectively. Order (group A or B) was not significant $(P=0 \cdot 98)$. Inclusion of body weight and age did not change the effect estimate for UIE $(P=0.19$ and $P=0.38$, respectively).

To assess the repeatability of UIE after consuming the same food, we compared the first and second soya milk intake for group A (Fig. 3) and the first and second miso soup intake for group B (Fig. 4). There was no significant difference in UIE between first and second intake for group $A$ or for group B ( $P=0.65$ and $P=0.81$, respectively).

Equol excretion was observed in seven of our twenty-one (33\%) participants. Three of the seven women showed equol excretion on all feeding days, three only on the initial feeding day, and one on feeding days 2 and 4 . We observed no significant difference in UIE between soya milk and miso

Table 1. Characteristics of the study population

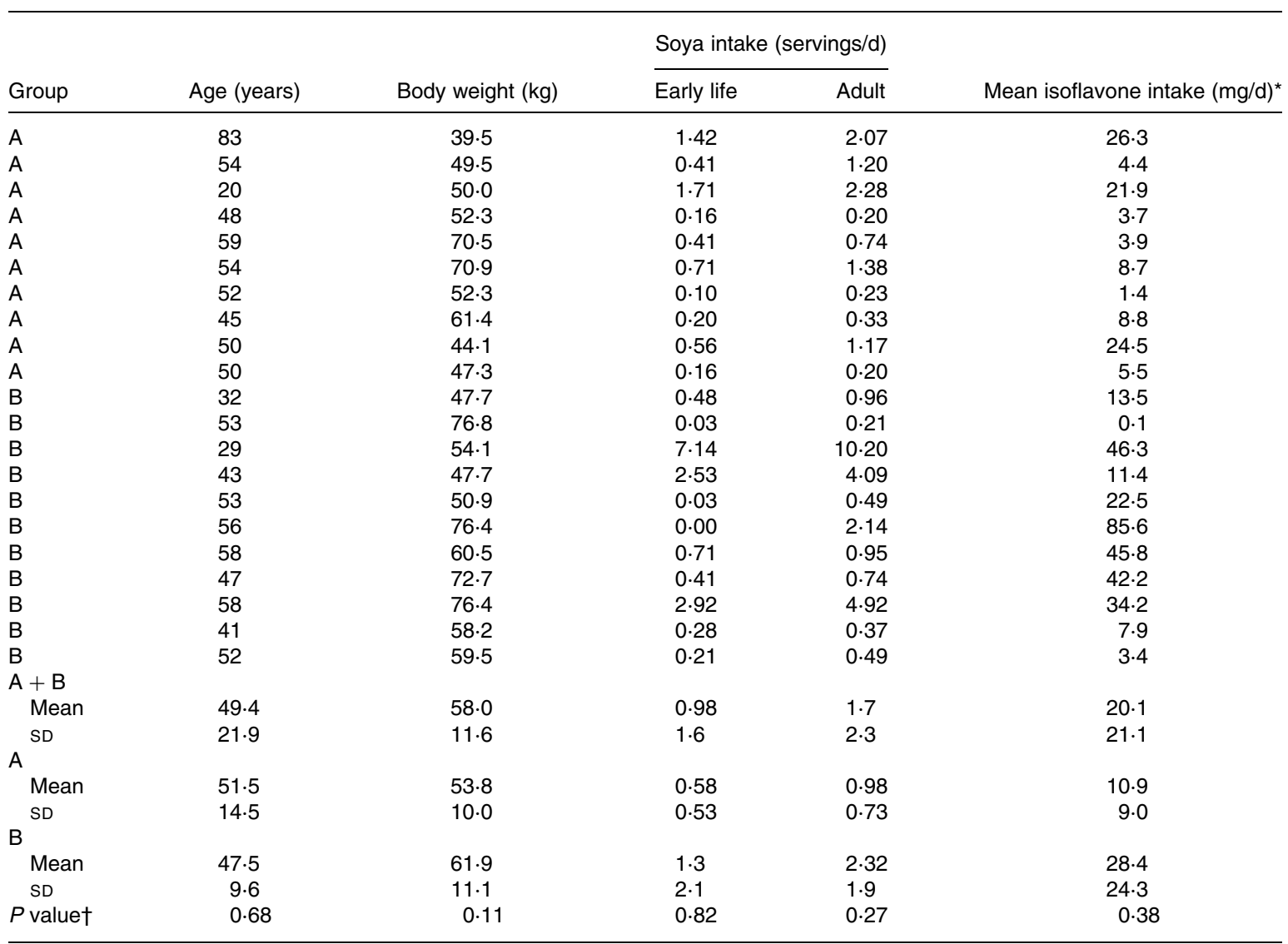

* Self-reported intake during the previous 12 months according to a FFQ.

$\dagger$ Two-sample $t$ tests using log-transformed values for difference between groups A and B. 


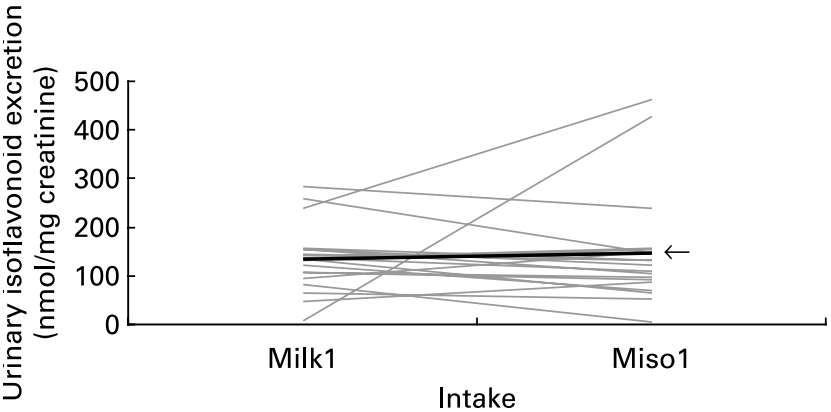

Fig. 1. Urinary isoflavonoid excretion on days 2 and 4 comparing first soya milk (Milk1) and miso soup (Miso1) intake. Each line represents one of the twenty-one Japanese-American women. $\leftarrow$, Mean.

soup for the seven equol producers $(P=0 \cdot 88)$. Based on the mixed model, there was no difference in overall UIE between equol producers and non-producers $(P=0 \cdot 87)$.

\section{Discussion}

In this small intervention study among twenty-one women with Japanese ancestry, we observed no significant difference in overnight UIE after intake of one serving of soya milk or miso soup with equivalent isoflavone content. Expressing UIE as $\mathrm{nmol} / \mathrm{mg}$ creatinine or as $\mathrm{nmol} / \mathrm{h}$ gave similar $P$ values ${ }^{(32)}$ and adjustment for potential confounders did not change the result. For the majority of subjects, the UIE did not vary by food (Fig. 1). The results are contrary to our expectation of a higher UIE for miso soup that contains readily available aglucones, whereas the isoflavones in soya milk require hydrolysis prior to uptake. Repeated intake of the same food on different days showed high reproducibility within subjects. There was also no difference in UIE among the subgroup of seven equol producers or between them and the non-equol producers. The fact that one-third of the participants excreted equol agreed with previous reports for Caucasians ${ }^{(11)}$ but is low for persons of Asian ancestry ${ }^{(33)}$.

The present results agree with some previous reports ${ }^{(21-23,34,35)}$, but they are in conflict with others ${ }^{(18-20,36,37)}$. Urinary isoflavonoid recovery did not differ significantly after a meal of cooked soyabeans, tofu, texturized vegetable protein or tempeh $^{(34)}$ between subjects who ingested soyabean isoflavone glycosides or red clover isoflavone aglycones ${ }^{(35)}$, and in a

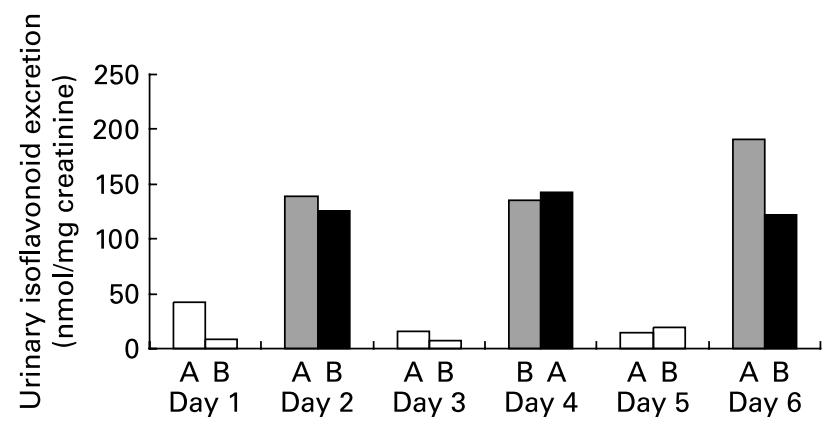

Fig. 2. Urinary isoflavonoid excretion before (days 1, 3 and 5; $\square$ ) and after soya food intake (days 2, 4 and 6; $\square$, soya milk; $\mathbf{\square}$, miso soup) in twenty-one Japanese-American women in two groups (A and B). Values are medians.

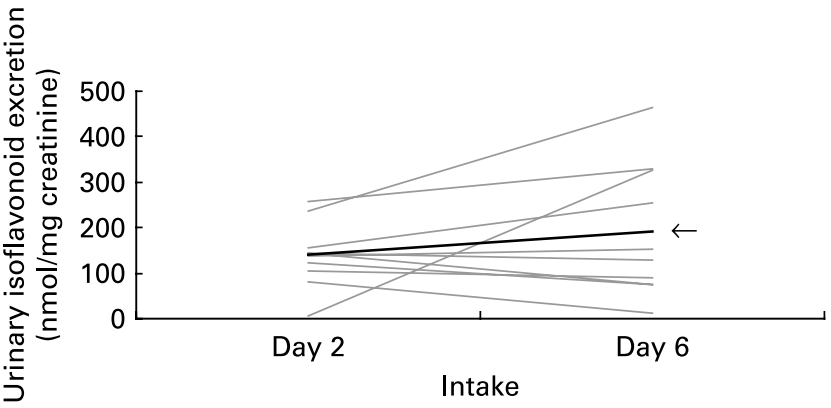

Fig. 3. Urinary isoflavonoid excretion after soya milk intake on days 2 and 6 . Each line represents one of the ten group A Japanese-American woman. $\leftarrow$, Mean.

cross-over trial that provided regular soya milk containing mostly glucosides and soya milk treated by probiotic bifidobacteria to produce aglucones ${ }^{(21)}$. Similarly, equivalent bioavailability was observed when isoflavones were given as glucosides as naturally present in soya drinks and after enzymatically hydrolysing these drinks to produce aglucones ${ }^{(23)}$, and bioavailability of isoflavones was similar among American women after consumption of aglucone or glucoside tablets ${ }^{(22)}$. A comparison of isolated aglucones and conjugated isoflavones also showed identical uptakes for both ${ }^{(8)}$. On the other hand, a randomized crossover trial described higher urinary isoflavonoid recovery after eating tempeh than soyabean pieces ${ }^{(18)}$, plasma concentrations over $24 \mathrm{~h}$ were higher after the administration of aglucone tablets than an equivalent glucoside preparation ${ }^{(19)}$, isoflavone aglycones were absorbed in greater amounts than their glycosides in a comparison of regular soya milk, fermented soya milk and $\beta$-glucosidase-treated soya milk ${ }^{(20)}$, and tempeh resulted in larger areas under the curve than textured vegetable protein ${ }^{(37)}$. Still another report demonstrated higher bioavailability of glucosides $v$. aglucones when the area under the plasma curve after oral dosage of $50 \mathrm{mg} \beta$-glucosides (daidzin, genistin) was compared to that after intake of the equivalent amount of aglycones (daidzein, genistein) $^{(36)}$.

Interventions that measured isoflavones in blood agree that the peak plasma level is achieved faster when aglucones as opposed to glucosides are consumed ${ }^{(19,20,36,37)}$. In the present study, it was not possible to assess the speed of isoflavone absorption during the first hours of consumption as described in studies that collected repeated blood samples ${ }^{(8,19,20,37)}$. However, the parallel pattern of plasma isoflavone levels

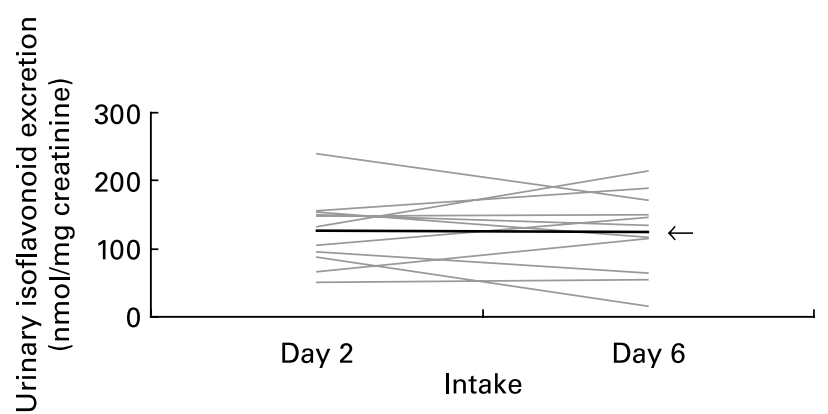

Fig. 4. Urinary isoflavonoid excretion after miso soup intake on days 2 and 6 . Each line represents one of the eleven group B Japanese-American woman. $\leftarrow$, Mean. 
and UIE when plotted as a function of time has been shown ${ }^{(8)}$. Published reports are consistent in that isoflavones in liquids rather than solids are absorbed faster, but cumulative uptake may be higher for solids ${ }^{(8,37,38)}$. Therefore, fermentation of soya foods leads to faster absorption and higher peak levels, but it appears likely that similar amounts of isoflavonoids from non-fermented products will become available over time.

The results of the present study have to be considered in light of several limitations, foremost the small sample size. Given the strong variation across subjects, the minimum difference by food that could have been detected with the present study, given a power of 0.80 , would have been $49 \mathrm{nmol} / \mathrm{mg}$ creatinine. Because all subjects were free-living, we were not able to verify the food intake and the exact times of soya consumption. In fact, it appears that at least one subject may not have consumed the soya milk at all (Fig. 1). Although our choice of two liquid soya foods eliminated bias due to the differential uptake according to the texture of the soya foods ${ }^{(37,38)}$, the women may have consumed the study foods as part of a regular meal whose composition may have affected the uptake of the isoflavones ${ }^{(37)}$. On the other hand, previous studies do not indicate a major effect of regular diet on the excretion of isoflavones ${ }^{(34,35)}$. Another issue was the lack of $24 \mathrm{~h}$ urine collections. Overnight urine samples represent the UIE that occurred over $10-12 \mathrm{~h}$. Therefore, we have no information on isoflavonoid excretion during the next day. However, the time frame in the present study covered approximately $70 \%$ of the total $\mathrm{UIE}^{(8)}$. Total excretion levels could have also been determined in $24 \mathrm{~h}$ urine samples but extensive periods of urine collection tend to result in low compliance.

The homogeneous ethnic background of the women with regular soya intake in the past was a strength because it reduced possible variation in UIE due to the development of specialized intestinal flora adapted to the breakdown of soya foods ${ }^{(24)}$. Individual differences in this cross-over study were minimized since the subjects served as their own controls and baseline UIE values were included in the statistical models. Results could be different in other ethnic groups who were not exposed to soya foods since childhood ${ }^{(24)}$. The wide age range in the present study, as well as unmeasured genetic and lifestyle factors, e.g. alcohol intake and exercise, may have also affected isoflavone uptake. Although UIE does not directly assess isoflavone absorption, the use of urine seemed adequate to estimate isoflavone bioavailability due to the high correlation of isoflavone appearance patterns in plasma and urine ${ }^{(8)}$, the general high correlation between plasma and urine values when samples are collected correctly $^{(32,39)}$, and the strong correlation between plasma and urine values as determined in different experimental settings ${ }^{(16,17)}$.

The interest in isoflavone uptake from fermented soya foods is based on the idea that the aglucones may be more readily bioavailable and more beneficial to human health ${ }^{(20)}$. Due to the continued ambiguity, the present study examined this question using an ethnically homogeneous population, a cross-over design, two liquid soya foods and a timed urine collection. The present results do not support the idea that the consumption of fermented soya foods results in higher isoflavonoid exposure than the intake of unfermented soya foods. Future studies with repeated blood and/or urine collections over more than $24 \mathrm{~h}$ among a larger study population are necessary to assess the speed of isoflavonoid uptake. At this time, the overall evidence does not justify nutritional recommendations that favour fermented soya foods as long as the intestinal microflora is capable of hydrolysing the isoflavone glucosides from unfermented soya foods.

\section{Acknowledgements}

We are grateful to the Meiji-Yasuda Foundation for supporting this intervention, the study participants, and Laurie Custer for isoflavonoid analysis of the urine specimens. Thanks to Beth Hopping for her help with the manuscript. Support by NCI (CA71789) is appreciated. None of the authors had a conflict of interest related to this project.

\section{References}

1. Barnes S, Boersma B, Patel R, Kirk M, Darley-Usmar VM, Kim $\mathrm{H} \& \mathrm{Xu}$ J (2000) Isoflavonoids and chronic disease: mechanisms of action. Biofactors 12, 209-215.

2. Adlercreutz H (2002) Phyto-oestrogens and cancer. Lancet Oncol 3, 364-373.

3. Barnes S, Peterson G, Grubbs C \& Setchell K (1994) Potential role of dietary isoflavones in the prevention of cancer. Adv Exp Med Biol 354, 135-147.

4. Franke AA, Hankin JH, Yu MC, Maskarinec G, Low SH \& Custer LJ (1999) Isoflavone levels in soy foods consumed by multiethnic populations in Singapore and Hawaii. J Agric Food Chem 47, 977-986.

5. Xu X, Harris KS, Wang HJ, Murphy PA \& Hendrich S (1995) Bioavailability of soybean isoflavones depends upon gut microflora in women. J Nutr 125, 2307-2315.

6. Setchell KD (2000) Absorption and metabolism of soy isoflavones - from food to dietary supplements and adults to infants. J Nutr 130, 654S-655S.

7. Setchell KD, Brown NM, Zimmer-Nechemias L, Brashear WT, Wolfe BE, Kirschner AS \& Heubi JE (2002) Evidence for lack of absorption of soy isoflavone glycosides in humans, supporting the crucial role of intestinal metabolism for bioavailability. Am J Clin Nutr 76, 447-453.

8. Franke AA, Custer L \& Hundahl S (2004) Determinants for urinary and plasma isoflavones in humans after soy intake. Nutr Cancer 50, 141-154.

9. Atkinson C, Frankenfeld CL \& Lampe JW (2005) Gut bacterial metabolism of the soy isoflavone daidzein: exploring the relevance to human health. Exp Biol Med (Maywood) 230, 155-170.

10. Axelson M, Sjovall J, Gustafsson BE \& Setchell KD (1984) Soya - a dietary source of the non-steroidal oestrogen equol in man and animals. $J$ Endocrinol 102, 49-56.

11. Setchell KD, Brown NM \& Lydeking-Olsen E (2002) The clinical importance of the metabolite equol - a clue to the effectiveness of soy and its isoflavones. J Nutr 132, 3577-3584.

12. Lampe JW, Gustafson DR, Hutchins AM, Martini MC, Li S, Wahala K, Grandits GA, Potter JD \& Slavin JL (1999) Urinary isoflavonoid and lignan excretion on a Western diet: relation to soy, vegetable, and fruit intake. Cancer Epidemiol Biomarkers Prev 8, 699-707.

13. Rowland IR, Wiseman H, Sanders TA, Adlercreutz H \& Bowey EA (2000) Interindividual variation in metabolism of soy isoflavones and lignans: influence of habitual diet on equol production by the gut microflora. Nutr Cancer 36, 27-32.

14. Seow A, Shi CH, Franke AA, Hankin H, Lee HP \& Yu MC (1998) Isoflavonoid levels in spot urine predict frequency of dietary soy 
intake in a population-based sample of middle-aged Chinese in Singapore. Cancer Epidemiol Biomarkers Prev 7, 135-140.

15. Maskarinec G, Singh S, Meng L \& Franke AA (1998) Dietary soy intake and urinary isoflavone excretion among women from a multiethnic population. Cancer Epidemiol Biomarkers Prev 7, 613-619.

16. Grace PB, Taylor JI, Low YL, et al. (2004) Phytoestrogen concentrations in serum and spot urine as biomarkers for dietary phytoestrogen intake and their relation to breast cancer risk in European prospective investigation of cancer and nutrition norfolk. Cancer Epidemiol Biomarkers Prev 13, 698-708.

17. Ritchie MR, Morton MS, Deighton N, Blake A \& Cummings JH (2004) Plasma and urinary phyto-oestrogens as biomarkers of intake: validation by duplicate diet analysis. BrJ Nutr 91, 447-457.

18. Hutchins AM, Slavin JL \& Lampe JW (1995) Urinary isoflavonoid phytoestrogen and lignan excretion after consumption of fermented and unfermented soy products. J Am Diet Assoc 95, $545-551$

19. Izumi T, Piskula MK, Osawa S, Obata A, Tobe K, Saito M, Kataoka S, Kubota Y \& Kikuchi M (2000) Soy isoflavone aglycones are absorbed faster and in higher amounts than their glucosides in humans. J Nutr 130, 1695-1699.

20. Kano M, Takayanagi T, Harada K, Sawada S \& Ishikawa F (2006) Bioavailability of isoflavones after ingestion of soy beverages in healthy adults. $J$ Nutr 136, 2291-2296.

21. Tsangalis D, Wilcox G, Shah NP \& Stojanovska L (2005) Bioavailability of isoflavone phytoestrogens in postmenopausal women consuming soya milk fermented with probiotic bifidobacteria. Br J Nutr 93, 867-877.

22. Zubik L \& Meydani M (2003) Bioavailability of soybean isoflavones from aglycone and glucoside forms in American women. Am J Clin Nutr 77, 1459-1465.

23. Richelle M, Pridmore-Merten S, Bodenstab S, Enslen M \& Offord EA (2002) Hydrolysis of isoflavone glycosides to aglycones by beta-glycosidase does not alter plasma and urine isoflavone pharmacokinetics in postmenopausal women. $J$ Nutr 132, 2587-2592.

24. Song KB, Atkinson C, Frankenfeld CL, Jokela T, Wahala K, Thomas WK \& Lampe JW (2006) Prevalence of daidzein-metabolizing phenotypes differs between Caucasian and Korean American women and girls. J Nutr 136, 1347-1351.

25. Williams AE, Maskarinec G, Hebshi S, Oshiro C, Murphy S \& Franke AA (2003) Validation of a soy questionnaire with repeated dietary recalls and urinary isoflavone assessments over one year. Nutr Cancer 47, 118-125.

26. Maskarinec G, Takata Y, Franke AA, Williams AE \& Murphy SP (2004) A 2-year soy intervention in premenopausal women does not change mammographic densities. J Nutr 134, 3089-3094.
27. Franke AA, Custer L, Wilkens L, LeMarchand L, Goodman MT \& Kolonel LN (2002) LC/PDA/MS analysis of dietary phytoestrogens from human urine and blood. J Chromatogr B 777, 43-57.

28. Blair RM, Appt SE, Franke AA \& Clarkson TB (2003) Treatment with antibiotics reduces plasma equol concentration in cynomolgus monkeys (Macaca fascicularis). J Nutr 133, 2262-2267.

29. Franke AA, Custer LJ, Wang W \& Shi SJ (1998) HPLC analysis of isoflavonoids and other phenolic agents from foods and from human fluids. Proc Soc Exp Biol Med 217, 263-273.

30. Dai Q, Franke AA, Yu H, Shu XO, Jin F, Hebert JR, Custer LJ, Gao YT \& Zheng W (2003) Urinary phytoestrogen excretion and breast cancer risk: evaluating potential effect modifiers, endogenous estrogens and anthropometrics. Cancer Epidemiol Biomarkers Prev 12, 497-502.

31. Littell RC, Milliken GA, Stroup WW \& Wolfinger RD (1996) SAS System for Mixed Models. Cary, NC: SAS Institute.

32. Franke AA, Halm BM, Custer LJ, Tatsumura Y \& Hebshi S (2006) Isoflavones in breastfed infants after mothers consume soy. Am J Clin Nutr 84, 406-413.

33. Akaza H, Miyanaga N, Takashima N, et al. (2004) Comparisons of percent equol producers between prostate cancer patients and controls: case-controlled studies of isoflavones in Japanese, Korean and American residents. Jpn J Clin Oncol 34, 86-89.

34. Xu X, Wang HJ, Murphy PA \& Hendrich S (2000) Neither background diet nor type of soy food affects short-term isoflavone bioavailability in women. $J$ Nutr 130, 798-801.

35. Tsunoda N, Pomeroy S \& Nestel P (2002) Absorption in humans of isoflavones from soy and red clover is similar. J Nutr 132, 2199-2201.

36. Setchell KD, Brown NM, Desai P, Zimmer-Nechemias L, Wolfe BE, Brashear WT, Kirschner AS, Cassidy A \& Heubi JE (2001) Bioavailability of pure isoflavones in healthy humans and analysis of commercial soy isoflavone supplements. J Nutr 131, 1362S-1375S.

37. Cassidy A, Brown JE, Hawdon A, Faughnan MS, King LJ, Millward J, Zimmer-Nechemias L, Wolfe B \& Setchell KD (2006) Factors affecting the bioavailability of soy isoflavones in humans after ingestion of physiologically relevant levels from different soy foods. J Nutr 136, 45-51.

38. de Pascual-Teresa S, Hallund J, Talbot D, Schroot J, Williams CM, Bugel S \& Cassidy A (2006) Absorption of isoflavones in humans: effects of food matrix and processing. J Nutr Biochem 17, 257-264.

39. Fanti P, Asmis R, Stephenson TJ, Sawaya BP \& Franke AA (2006) Positive effect of dietary soy in ESRD patients with systemic inflammation - correlation between blood levels of the soy isoflavones and the acute-phase reactants. Nephrol Dial Transplant 21, 2239-2246. 